\title{
High fat diet-induced obesity causes a reduction in brain tyrosine hydroxylase levels and non-motor features in rats through metabolic dysfunction, neuroinflammation and oxidative stress
}

\author{
Aline Bittencourt, Pedro Ozorio Brum, Camila Tiefensee Ribeiro, Juciano \\ Gasparotto, Rafael Calixto Bortolin, Amanda Rodrigues de Vargas, Luana \\ Heimfarth, Roberto Farina de Almeida, José Claudio Fonseca Moreira, Jade de \\ Oliveira, Daniel Pens Gelain
}

\begin{abstract}
Obesity is a health problem that has been associated with neuroinflammation, decreased cognitive functions and development of neurodegenerative diseases. Parkinson's disease (PD) is a chronic neurodegenerative condition characterized by motor and non-motor abnormalities, increased brain inflammation, $\alpha$-synuclein protein aggregation and dopaminergic neuron loss that is associated with decreased levels of tyrosine hydroxylase $(\mathrm{TH})$ in the brain. Diet-induced obesity is a global epidemic and its role as a risk factor for PD is not clear. Herein, we showed that 25 weeks on a high-fat diet (HFD) promotes significant alterations in the nigrostriatal axis of Wistar rats. Obesity induced by HFD exposure caused a reduction in $\mathrm{TH}$ levels and increased $\mathrm{TH}$ phosphorylation at serine 40 in the ventral tegmental area. These effects were associated with insulin resistance, increased tumor necrosis factor- $\alpha$ levels, oxidative stress, astrogliosis and microglia activation. No difference was detected in the levels of $\alpha$-synuclein. Obesity also induced impairment of locomotor activity, total mobility and anxiety-related behaviors that were identified in the open-field and light/dark tasks. There were no changes in motor coordination or memory. Together, these data suggest that the reduction of $\mathrm{TH}$ levels in the nigrostriatal axis occurs through an $\alpha$-synucleinindependent pathway and can be attributed to brain inflammation, oxidative/nitrosative stress and metabolic disorders induced by obesity.
\end{abstract}

\section{Palabras clave}

Obesity,Parkinson's disease,Tyrosine hydroxylase, Inflammation, Locomotion, Anxiety, Neuroinflammation, High-fat diet 